\title{
Immunohistological detection of Legionella pneumophila in lung sections
}

\author{
J M THEAKER, J O'H TOBIN,* S E C JONES, P KIRKPATRICK, M I VINA, \\ K A FLEMING
}

From the Nuffield Department of Pathology, and the *Department of Virology, John Radcliffe Hospital, Oxford

SUMMARY Immunohistology was used for the detection of Legionella pneumophila serogroup I in necropsy tissue. Study of pneumonic lung from the recent Stafford outbreak has shown that this technique has a high sensitivity. A retrospective postmortem examination showed that $L$ pneumophila serogroup 1 was an unusual cause of pneumonia in Oxfordshire during the study period. $L$ pneumophila serogroup 1 can be successfully subgrouped, using a panel of monoclonal antibodies on formalin fixed paraffin embedded sections. Immunohistological methods have a potentially useful role in the diagnosis of Legionellosis at postmortem examination and in the epidemiological investigation of individual cases and outbreaks.

The recognition of the agents responsible for infectious disease usually depends on culture of the organism, followed by examination of its morphological and biochemical properties. Some organisms, however, are difficult to grow, and on occasions suitable material may not be available for culture. Immunohistological techniques permit the sensitive and specific detection of antigenic determinants and already have an established use in the identification of micro-organisms, both in fresh ${ }^{1}$ and fixed tissue sections. ${ }^{23}$

Legionella pneumophila is a cocco-bacillary bacterium that can cause pneumonia both sporadically and in epidemics. ${ }^{4}$ It may be difficult to culture from pathological specimens, and correct identification in tissue sections with the silver impregnation Dieterle stain is often impossible, especially in lung where carbon and other particulate material can cause confusion. Accordingly, its rapid diagnosis is now often made by immunofluorescence examination of bronchial washings or sputum. ${ }^{4}$ On occasion, however, (when the organism is not suspected), such fresh material is not available and the diagnosis cannot be confirmed. In view of this we investigated the possibility of detecting $L$ pneumophila by immunohistology on formalin fixed necropsy lung sections to determine whether this technique may be a useful adjunct in the diagnosis of Legionella pneumonia. We assessed the sensitivity of the method by postmortem examination of lung sections from patients who died during

Accepted for publication 25 August 1986 the recent Legionella outbreak at Stafford, ${ }^{5}$ and we also determined whether the organism could be subgrouped in tissue sections using a panel of monoclonal antibodies. In addition, we retrospectively investigated the incidence of Legionella pneumonia at postmortem examination using this technique.

\section{Material and methods}

Formalin fixed paraffin embedded lung sections were kindly provided from 28 necropsy cases of suspected Legionella pneumonia from the Stafford outbreak. Sections were also obtained from the files of this hospital from 70 consecutive necropsy cases of pneumonia, both hospital and community acquired, in which $L$ pneumophila had not been suspected.

These were stained by an indirect immunoperoxidase technique ${ }^{6}$ after endogenous peroxidase activity was blocked with hydrogen peroxide and after trypsin treatment of the sections.

In the first stage a rabbit polyclonal anti- $L$ pneumophila serogroup 1 antibody was used at a dilution of $1 / 200$. This is the antiserum used routinely in the immunofluorescence examination of sputum and bronchial washings in Oxford (supplied by Dr AG Taylor, Division of Microbiological Reagents and Quality Control, Central Public Health Laboratory, London) and has proved reliable in clinical testing over seven years, detecting no organisms other than $L$ pneumophila.

The appropriate test dilution used was determined by staining necropsy lung sections from three cases of known $L$ pneumophila serogroup 1 pneumonia, using 
serial dilutions of the antiserum. The three cases gave consistent and similar staining. A negative control was performed by substitution of the anti-Legionella antibody by non-immune rabbit serum or by omission of the first stage antibody.

Six of the Stafford cases were further studied. Paraffin sections were dewaxed, fixed in acetone for five minutes, and then treated for one hour at $37^{\circ} \mathrm{C}$ with $20 \mu \mathrm{l}$ of one of the nine monoclonal antibodies that have been used to subgroup members of $L$ pneumophila serogroup $1 .^{7}$ After washing twice for five minutes in phosphate buffered saline (PBS) $20 \mu \mathrm{l}$ of antimouse fluorescent conjugate was applied for 25 minutes. After further washing in PBS and drying each section was examined for organisms with a Leitz fluorescence microscope, using incident light illumination. The results of postmortem lung culture for $L$ pneumophila from the Stafford patients were kindly made available by Dr JGP Hutchinson, Public Health Laboratory Service, Birmingham.

\section{Results}

Eleven of the 28 cases from Stafford showed positive staining with the rabbit anti-Legionella serum (figs 1 2 , and 3). Several patterns were seen. Firstly, organisms could be seen within neutrophil polymorphs and macrophages, either as single bacteria or as small clusters. Secondly, a large globular area of strong staining could be seen in scattered macrophages, which, on oil immersion microscopy, showed a coarse granular pattern, probably representing a colony of intracellular bacteria. Thirdly, in the strongest staining case there was a diffuse, weak staining of the alveolar exudate and cytoplasm of phagocytes. This may represent specific staining of bacterial anti-

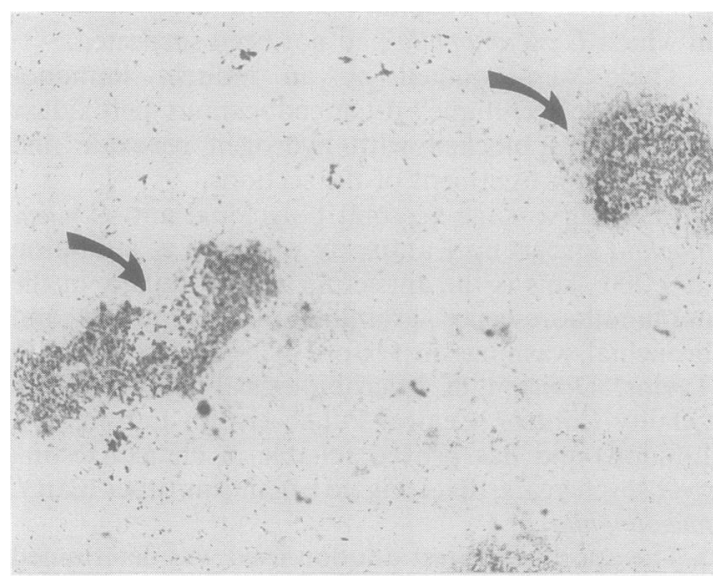

Fig 1 Low power view of pneumonic lung showing patchy staining for Legionella (arrows) (Peroxidase.) $\times 50$.
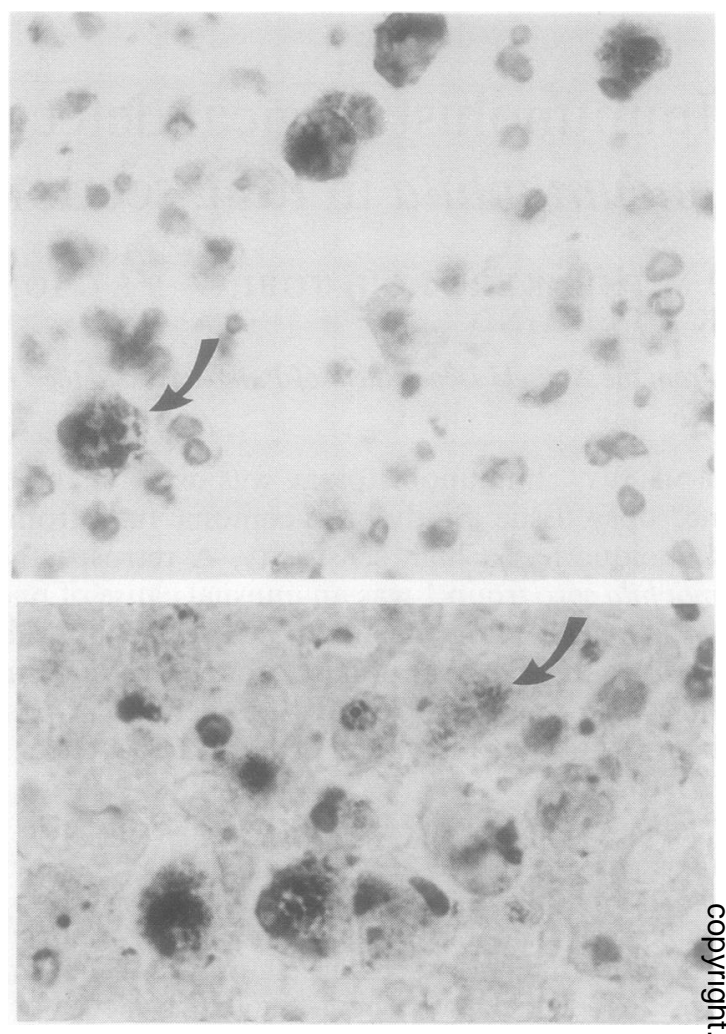

Figs 2 and 3 Clusters (arrows) and larger colonies of organisms present mainly within macrophages. (Peroxidase.) $\times 1200$.

genic debris. In all cases, however, staining was patchy, with a few cases having only one or several small detectable foci.

In all cases no staining was noted with the negative control, and there was no difficulty distinguishing the positive staining from background carbon, haemosiderin, or other particulate debris.

The table shows the relation between Legionella staining and the histological features seen in the stained sections, and also indicates, for comparison, the results of culture. Most cases of acute pneumonia (eight of 11), and of organising pneumonia (three of six) were positive on immunohistology. By contrast, sections of lung tissue from the 11 cases, which showed severe changes, but which were not predominantly pneumonic (shock lung, infarction, alveolar haemorrhage, thromboembolism), were negative on immunohistology.

Of the nine culture positive cases, three were negative by immunohistology on the available lung sections. Of these three cases, one showed the histological features of shock lung, one organisation of pneumonia, and only one showed acute pneumonic 
Table Immunoperoxidase staining of necropsy lung sections from Stafford for Legionella pneumophila serogroup I* $^{*}$

\begin{tabular}{lccc}
\hline Dominant histological features & Total & No positive for Legionella & No negative for Legionella \\
\hline Severe pneumonia, early or minor organisation & $11(6)$ & $8(5)$ & $3(1)$ \\
Predominantly organisation with focal active pneumonia & $6(2)$ & $3(1)$ & $3(1)$ \\
Major pathology other than pneumonia & $11(1)$ & 0 & $11(1)$ \\
& $28(9)$ & $11(6)$ & $17(3)$ \\
\hline
\end{tabular}

*Numbers in parentheses represent cases confirmed by culture.

consolidation. Conversely, immunohistology detected five cases which showed Legionella staining, but which were negative on culture.

Further sections from six of the 11 positive cases were studied by immunofluorescence using a panel of nine monoclonal anti-Legionella antibodies. In all cases bacteria were stained by the same two antibodies alone, identifying them as subgroup Pontiac 1a, the same subgroup as the isolates from Stafford. ${ }^{5}$

Retrospective immunohistological examination of lung tissue from 70 consecutive cases shown to have pneumonia at postmortem examination was negative. One of these was a known case of $L$ bozemanii pneumonia, but this was negative to testing with the antibody to $L$ pneumophila serogroup I, although it proved positive using a rabbit polyclonal antibody against $L$ bozemanii.

\section{Discussion}

Immunofluorescence examination of bronchial washings, sputum, or homogenised lung tissue is a well established method for the diagnosis of Legionella. ${ }^{4}$ Indeed, this is generally more sensitive than culture, possibly due to difficulties with overgrowth of other bacteria, or loss of viability, either due to drug treatment or during storage and handling. Furthermore, these immunofluorescence methods offer the advantage of a more rapid diagnosis than culture. Therefore, it has been recommended that culture should be supplemented by immunofluorescence when the diagnosis of legionellosis is considered. ${ }^{8}$

Although the detection by immunofluorescence of the organism in formalin fixed paraffin embedded lung sections has been successful, ${ }^{9}$ it is well recognised that immunohistochemical techniques have several advantages compared with immunofluorescence, including direct visualisation of the stained antigenic material and the morphological features of the tissue. Accordingly, we investigated the use of an indirect immunoperoxidase technique in the diagnosis of Legionella pneumonia. The method gave clear and reproducible staining of colonies and single bacteria, and, on occasion, a more diffuse antigenic background.

The study of the lung tissue from the Stafford outbreak permitted an assessment of the sensitivity of this technique. We detected Legionella by positive staining in 11 of 28 cases compared with nine culture positive cases. There was a close correlation with the histopathological nature of the lung lesion, as eight of the 11 cases with acute pneumonic consolidation showed positive staining for Legionella compared with six by culture. Only one case of immunohistologically negative acute pneumonia was positive on culture. Of the six cases of organising pneumonia examined, we detected staining for Legionella in three. As staining was strikingly patchy in some cases, investigation of additional blocks or of further levels might have increased the detection rate. Not surprisingly, none of the 11 cases, in which the lung section showed changes that may have complicated the severe pneumonic illness, but not pneumonia itself, was positive for Legionella. From these results we suggest that immunohistochemical identification of Legionella is at least as sensitive as culture, particularly if multiple blocks from the areas of greatest pneumonic consolidation are investigated.

This technique has the same advantages as immunofluorescence compared with culture - that is, equal or greater sensitivity, and, potentially a more rapid diagnosis. It also, however, has the unique advantage of permitting the retrospective detection of Legionella, if fresh lung tissue had not been sent for culture and if formalised lung tissue is no longer available for direct immunofluorescence on lung scrapings. Furthermore, the method could also be applied to lung biopsy specimens, in cases showing pneumonia of undetermined cause.

It has recently been shown that $L$ pneumophila serogroup 1 can be reliably further subgrouped using a panel of monoclonal antibodies against a range of surface antigens. ${ }^{710}$ In the present study we have shown that this method of subgrouping can be successfully performed on formalin fixed paraffin embedded lung sections. This could have considerable epidemiological importance in tracing the environmental source of the infecting Legionella, especially if several potential sources with different Legionella subgroups have been identified, and no isolates of Legionella have been obtained from the patients.

As the reported incidence of Legionella as a cause of pneumonia ranges from 1 to $20 \%{ }^{11}$ (depending on the population examined, methods used, and the 
the population examined, methods used, and the region studied), we applied this technique to 70 unselected and consecutive cases of pneumonia detected at postmortem examination. We failed to detect $L$ pneumophila serogroup 1 in any. As our study of the Stafford cases has shown that this technique has a high sensitivity, especially in cases of acute pneumonic consolidation, we conclude that $L$ pneumophila serogroup 1 (the commonest serogroup causing Legionella pneumonia ${ }^{12}$ ) has been an unusual cause of sporadic pneumonia, both in hospital and community acquired cases in the Oxford region.

We thank Dr FJ Pick, Staffordshire General Infirmary, for providing pathological sections, Dr JGP Hutchison, Public Health Laboratory Service, Birmingham, for culture results, Dr JB Kurtz, Department of Virology, Oxford, for help throughout this project, and Miss Lesley Watts for typing the manuscript.

\section{References}

1 Gardner PS, McQuillin J. Rapid virus diagnosis. Application of immunofluorescence. London: Butterworths, 1974.

2 Burns J. Immunoperoxidase localisation of hepatitis B antigen (HB) in formalin-paraffin processed liver tissue. Histochemistry 1975;44:133-5.
3 Woodruff JD, Braun L, Cavalieri R, Gupta P, Pass F, Shah KV. Immunologic identification of papillomavirus antigen in condlyoma tissues from the female genital tract. Obstet Gynecol 1980;56:727-32.

4 Blackmon JA, Chandler FW, Cherry WB, et al. Legionellosis: review article. Am J Pathol 1981;103:429-65.

5 First report of the committee of inquiry into the outbreak of Legionnaires Disease in Stafford in April 1985. London: HMSO (Cmnd 9772), 1986.

6 Gatter KC, Falini B, Mason DY. The use of monoclonal antibodies in histopathological diagnosis. Rec Adv Histopathol 1984;12:35-67.

7 Watkins ID, Tobin J O'H, Dennis PJ, Brown W, Newnham R, Kurtz JB. Legionella pneumophila serogroup 1 subgrouping by monoclonal antibodies-an epidemiological tool. $J$ Hyg Camb 1985;95:211-6.

8 Edelstein PH, Meyer RD, Finegold SM. Laboratory diagnosis of Legionnaire's disease. Am Rev Respir Dis 1980;121:317-27.

9 Cherry WB, Pittman B, Harris PP, et al. Detection of Legionnaires disease bacteria by direct immunofluorescent staining. $J$ Clin Microbiol 1978;8:329-38.

10 Joly JR, McKinney RM, Tobin JO, Bibb WF, Watkins ID, Ramsay D. Development of a standardised subgrouping scheme for Legionella pneumophila serogroup 1 using monoclonal antibodies. J Clin Microbiol 1986;23:768-71.

11 Anonymous. How common is Legionnaires disease? [Editorial] Lancet 1983;i:103-4.

12 Reingold AL, Thomason BM, Brake BJ, Thacker L, Wilkinson HW, Kuritsky JN. Legionella pneumophila in the United States: the distribution of serogroups and species causing human pneumonia. J Infect Dis 1984;149:819.

Requests for reprints to: Dr JM Theaker, NuffieldO Department of Pathology, John Radcliffe Hospital, Level 4, Headington, Oxford OX3 9DU, England. 